\title{
On Complex Contact Similarity Manifolds
}

\author{
Yoshinobu Kamishima ${ }^{1} \&$ Akira Tanaka $^{2}$ \\ ${ }^{1}$ Department of Mathematics, Tokyo Metropolitan University, Japan \\ 2 Department of Mathematics and Information of Sciences, Tokyo Metropolitan University, Japan \\ Correspondence: Akira Tanaka, Department of Mathematics and Information of Sciences, Tokyo Metropolitan \\ University, Japan. E-mail: tanaka-akira@ed.tmu.ac.jp
}

Received: July 14, 2013 Accepted: August 27, 2013 Online Published: September 29, 2013

doi:10.5539/jmr.v5n4p1 URL: http://dx.doi.org/10.5539/jmr.v5n4p1

\begin{abstract}
We shall construct complex contact similarity manifolds. Among them there exists a complex contact infranilmanifold $\mathcal{L} / \Gamma$ which is a holomorphic torus fiber space over a quaternionic euclidean orbifold. Specifically taking a connected sum of $\mathcal{L} / \Gamma$ with the complex projective space $\mathbb{C P}^{2 n+1}$, we prove that the connected sum admits a complex contact structure. Our examples of complex contact manifolds are different from those known previously as complex Boothby-Wang fibration (Foreman, 2000) or the twistor fibration (Salamon, 1989).
\end{abstract}

Keywords: complex contact structure, Sasakian 3-structure, Twistor space, complex Boothby-Wang fibration, Infranil-manifold, Quaternionic Kähler manifold

2010 Mathematics Subject Classification: 53D10, 57S25, 32Q55

\section{Introduction}

There is a construction of three different types of complex contact structure. Given a $4 n$-dimensional quaternionic Kähler manifold $N$ of nonzero scalar curvature, the twistor construction produces a complex contact manifold $M$ which is the total space of a fibration: $S^{2} \rightarrow M \rightarrow N$ (cf. Salamon, 1989; Wolf, 1965). Similarly, a quaternionic Kähler manifold $N^{4 n}$ of positive (resp. negative) scalar curvature induces a Sasakian 3-structure (resp. pseudoSasakian 3-structure) on the total space $M^{4 n+3}$ of the principal fibration: $S^{3} \rightarrow M \rightarrow N$. By taking a circle $S^{1}$ from $S^{3}$, the total space $M / S^{1}$ of the quotient bundle $S^{2} \rightarrow M / S^{1} \rightarrow N$ admits a complex contact structure. (See Ishihara \& Konishi, 1979; Moroianu \& Semmelmann, 1996; Tanno, 1996). However, these constructions cannot produce complex contact manifolds for quaternionic Kähler manifolds of vanishing scalar curvature. On the other hand, if $N^{4 n}$ is a complex symplectic manifold with a complex symplectic form $\Omega=\Omega_{1}+\mathbf{i} \Omega_{2}$ such that $\left[\Omega_{i}\right] \in H^{2}(N ; \mathbb{Z})$ is an integral class $(i=1,2)$, then the complex Boothby-Wang fibration induces a compact complex contact manifold $M$ which has a connection bundle: $T^{2} \rightarrow M \rightarrow N$ (cf. Foreman, 2000; Blair, 2002). If $N^{4 n}$ happens to be a quaternionic Kähler manifold with vanishing scalar curvature, then we have a new example of compact complex manifold. In fact, Foreman (2000) shows that a complex nilmanifold $M$ which is the total space of a principal torus bundle over a complex torus $T_{\mathbb{C}}^{2 n}$ admits a complex contact structure. The universal covering $\tilde{M}$ is endowed with a complex nilpotent Lie group structure which is called generalized complex Heisenberg group in Foreman, 2000.

In this paper, we study complex contact transformation groups by taking into account this specific nilpotent Lie group. We verify this group from the viewpoint of geometric structure in Section 4. In fact the sphere $S^{4 n+3}$ admits a canonical quaternionic $C R$-structure. The sphere $S^{4 n+3}$ with one point $\infty$ removed is isomorphic to the $4 n+3$-dimensional quaternionic Heisenberg Lie group $\mathcal{M}$ as a quaternionic $C R$-structure. $\mathcal{M}$ has a central group extension: $1 \rightarrow \mathbb{R}^{3} \rightarrow \mathcal{M} \stackrel{p}{\longrightarrow} \mathbb{H}^{n} \rightarrow 1$ where $\mathbb{R}^{3}=\operatorname{Im} \mathbb{H}$ is the imaginary part of the quaternion field $\mathbb{H}$. Taking a quotient of $\mathcal{M}$ by $\mathbb{R}(=\mathbb{R} \mathbf{i})$, we obtain a complex nilpotent Lie group $\mathcal{L}\left(=\mathcal{L}_{2 n+1}\right)$ which supports a holomorphic principal bundle $\mathbb{C} \rightarrow \mathcal{L} \stackrel{p}{\longrightarrow} \mathbb{C}^{2 n}$. The canonical quaternionic $C R$-structure on $S^{4 n+3}$ restricts a Carnot-Carathéodory structure $B$ to $\mathcal{M}$. Using this bundle $B$, a left invariant complex contact structure on $\mathcal{L}$ is obtained (cf. Alekseevsky \& Kamishima, 2008; Kamishima, 1999).

We are mainly interested in constructing examples of compact complex contact manifolds which are not known 
previously. Let $\operatorname{Sim}(\mathcal{L})$ be the group of complex contact similarity transformations. It is defined to be the semidirect product $\mathcal{L} \rtimes\left(\operatorname{Sp}(n) \cdot \mathbb{C}^{*}\right),\left(\mathbb{C}^{*}=S^{1} \times \mathbb{R}^{+}\right)$. The pair $(\operatorname{Sim}(\mathcal{L}), \mathcal{L})$ is said to be complex contact similarity geometry. A manifold $M$ locally modelled on this geometry is called a complex contact similarity manifold. Denote by $\operatorname{Aut}_{c c}(M)$ the group of complex contact transformations of $M$. We prove the following characterization of compact complex contact similarity manifolds in Section 2 (Compare Fried, 1980; Miner, 1991 for the related results in this direction).

Theorem A Let $M$ be a compact complex contact similarity manifold of complex dimension $2 n+1$. If $S^{1} \leq$ $\operatorname{Aut}_{c c}(M)$ acts on $M$ without fixed points, then $M$ is holomorphically diffeomorphic to a complex contact infranilmanifold $\mathcal{L} / \Gamma$ or a complex contact Hopf manifold $\mathcal{L}-\{0\} / \mathbb{Z}^{+}$diffeomorphic to $S^{1} \times S^{4 n+1}$. Here $\Gamma$ is a discrete cocompact subgroup in $\mathcal{L} \rtimes\left(\operatorname{Sp}(n) \cdot S^{1}\right)$ or $\mathbb{Z}^{+}$is an infinite cyclic subgroup of $\operatorname{Sp}(n) \cdot S^{1} \times \mathbb{R}^{+}$.

In Section 3, we can perform a connected sum of our complex contact infranil-manifolds $\mathcal{L} / \Gamma(\Gamma \leq \mathrm{E}(\mathcal{L}))$.

Theorem B The connected sum $\mathbb{C P}^{2 n+1} \# \mathcal{L} / \Gamma$ admits a complex contact structure.

By iteration of this procedure there exists a complex contact structure on the connected sum of a finite number of complex contact similarity manifolds and $\mathbb{C P}^{2 n+1}$ 's. These examples are different from those admitting $S^{2}$ (resp. $T^{2}$ )-fibrations

\section{Complex Contact Structure on the Nilpotent Group}

\subsection{Definition of Complex Contact Structure}

Recall that a complex contact structure on a complex manifold $M$ in complex dimension $2 n+1$ is a collection of local forms $\left\{U_{\alpha}, \omega_{\alpha}\right\}_{\alpha \in \Lambda}$ which satisfies that (1) $\cup_{\alpha \in \Lambda} U_{\alpha}=M$. (2) Each $\omega_{\alpha}$ is a holomorphic 1-form defined on $U_{\alpha}$. Then $\omega_{\alpha} \wedge\left(d \omega_{\alpha}\right)^{n} \neq 0$ on $U_{\alpha}$. (3) If $U_{\alpha} \cap U_{\beta} \neq \emptyset$, then there exists a nonzero holomorphic function $f_{\alpha \beta}$ on $U_{\alpha} \cap U_{\beta}$ such that $f_{\alpha \beta} \cdot \omega_{\alpha}=\omega_{\beta}$. Unlike contact structures on orientable smooth manifolds, it does not always exist a holomorphic 1-form globally defined on $M$. Note that if the first Chern class $c_{1}(M)$ vanishes, then there is a global existence of a complex contact form $\omega$ on $M$. (See Kobayashi, 1959; Lebrun, 1995).

Let $h: M \rightarrow M$ be a biholomorphism. Suppose that $h\left(U_{\alpha}\right) \cap U_{\beta} \neq \emptyset$ for some $\alpha, \beta \in \Lambda$. If there exists a holomorphic function $f_{\alpha \beta}$ on an open subset in $U_{\alpha}$ such that $h^{*} \omega_{\beta}=f_{\alpha \beta} \omega_{\alpha}$, then we call $h$ a complex contact transformation of $M$. Denote $\operatorname{Aut}_{c c}(M)$ the group of complex contact transformations. It is not necessarily a finite dimensional complex Lie group.

\subsection{The Iwasawa Nilpotent Lie Group $\mathcal{L}_{2 n+1}$}

Let $\mathcal{L}_{2 n+1}$ be the product $\mathbb{C}^{2 n+1}=\mathbb{C} \times \mathbb{C}^{2 n}$ with group law $(n \geq 1)$ :

$$
(x, z) \cdot(y, w)=\left(x+y+\sum_{i=1}^{n} z_{2 i-1} w_{2 i}-z_{2 i} w_{2 i-1}, z+w\right)
$$

where $z=\left(z_{1}, \ldots, z_{2 n}\right), w=\left(w_{1}, \ldots, w_{2 n}\right)$.

Put $\mathcal{L}=\mathcal{L}_{2 n+1}$. It is easy to see that $[(x, z),(y, w)]=\left(2 \sum_{i=1}^{n} z_{2 i-1} w_{2 i}-z_{2 i} w_{2 i-1}, 0\right)$ so $[\mathcal{L}, \mathcal{L}]=(\mathbb{C},(0, \ldots, 0))=\mathbb{C}$ is the center of $\mathcal{L}$. Thus there is a central group extension: $1 \rightarrow \mathbb{C} \rightarrow \mathcal{L}_{2 n+1} \longrightarrow \mathbb{C}^{2 n} \rightarrow 1$. It is easy to check that $\mathcal{L}_{3}$ is isomorphic to the Iwasawa group consisting of $3 \times 3$-upper triangular unipotent complex matrices.

Definition 2.1 A complex $2 n+1$-dimensional complex nilpotent Lie group $\mathcal{L}_{2 n+1}$ is said to be the Iwasawa Lie group.

See (Foreman, 2000, pp.193-195) for more general construction of this kind of Lie group.

\subsection{Construction of Complex Contact Structure on $\mathcal{L}_{2 n+1}$}

Choose a coordinate $\left(z_{0}, z_{1}, \ldots, z_{2 n}\right) \in \mathcal{L}_{2 n+1}$, we define a complex 1-form $\eta$ :

$$
\eta=d z_{0}-\left(\sum_{i=1}^{n} z_{2 i-1} \cdot d z_{2 i}-z_{2 i} \cdot d z_{2 i-1}\right)=d z_{0}-\left(z_{1}, \ldots, z_{2 n}\right) \mathrm{J}_{n}\left(\begin{array}{c}
d z_{1} \\
\vdots \\
d z_{2 n}
\end{array}\right)
$$


where $J_{n}=\left(\begin{array}{ccc}J & & \\ & \ddots & \\ & & J\end{array}\right)$ with $J=\left(\begin{array}{cc}0 & 1 \\ -1 & 0\end{array}\right)$.

Since $\eta \wedge(d \eta)^{n}$ is a non-vanishing form $2 n(-2)^{n} d z_{0} \wedge \cdots \wedge d z_{2 n}$ on $\mathcal{L}_{2 n+1}, \eta$ is a complex contact structure on $\mathcal{L}_{2 n+1}$ by Definition 2.1.

\subsection{Complex Contact Transformations}

Let hol $\left(\mathcal{L}_{2 n+1}\right)$ be the group of biholomorphic transformations of $\mathcal{L}=\mathcal{L}_{2 n+1}$. The group of complex contact transformations on $\mathcal{L}$ with respect to $\eta$ is denoted by

$$
\operatorname{hol}(\mathcal{L}, \eta)=\left\{f \in \operatorname{hol}(\mathcal{L}) \mid f^{*} \eta=\tau \cdot \eta\right\}
$$

where $\tau$ is a holomorphic function on $\mathcal{L}$.

Let $\operatorname{Sp}(n, \mathbb{C})=\left\{\left.A \in M(2 n, \mathbb{C})\right|^{t} A J_{n} A=J_{n}\right\}$ be the complex symplectic group. As $\operatorname{Sp}(n, \mathbb{C}) \cap \mathbb{C}^{*}=\{ \pm 1\}$, denote $\operatorname{Sp}(n, \mathbb{C}) \cdot \mathbb{C}^{*}=\operatorname{Sp}(n, \mathbb{C}) \times \mathbb{C}^{*} /\{ \pm 1\}$. Put

$$
\mathrm{A}(\mathcal{L})=\mathcal{L} \rtimes\left(\operatorname{Sp}(n, \mathbb{C}) \cdot \mathbb{C}^{*}\right)
$$

which forms a group as follows; write elements $\lambda \cdot A, \mu \cdot B \in \operatorname{Sp}(n, \mathbb{C}) \cdot \mathbb{C}^{*}$ for $A, B \in \operatorname{Sp}(n, \mathbb{C}), \lambda, \mu \in \mathbb{C}^{*}$. Let $(a, w),(b, z) \in \mathcal{L}$. Define

$$
((a, w), \lambda \cdot A) \cdot((b, z), \mu \cdot B)=\left(\left(a+\lambda^{2} b+{ }^{t} w \mathrm{~J}_{n}(\lambda A z), w+\lambda A z\right), \lambda \mu \cdot A B\right) .
$$

Here ${ }^{t} w J_{n}(\lambda A z)=\sum_{i=1}^{n} w_{2 i-1} \cdot(\lambda A z)_{2 i}-w_{2 i} \cdot(\lambda A z)_{2 i-1}$ as before.

Let $((a, w), \lambda \cdot A) \in \mathrm{A}(\mathcal{L}),\left(z_{0}, z\right) \in \mathcal{L}$. $\mathrm{A}(\mathcal{L})$ acts on $\mathcal{L}$ as

$$
((a, w), \lambda \cdot A) \cdot\left(z_{0}, z\right)=(a, w) \cdot\left(\lambda^{2} z_{0}, \lambda A z\right)=\left(a+\lambda^{2} z_{0}+{ }^{t} w J_{n}(\lambda A z), w+\lambda A z\right) .
$$

If $h=((b, w), \mu \cdot B) \in \mathrm{A}(\mathcal{L})$ is an element, then it is easy to see that

$$
h^{*} \eta=\mu^{2} \cdot \eta \text {. }
$$

Thus $\mathrm{A}(\mathcal{L})$ preserves the complex contact structure on $\mathcal{L}$ defined by $\eta$.

Let $\operatorname{Aff}\left(\mathbb{C}^{2 n+1}\right)=\mathbb{C}^{2 n+1} \rtimes \mathrm{GL}(2 n+1, \mathbb{C})$ be the complex affine group which is a subgroup of hol $(\mathcal{L})$ since $\mathcal{L}_{2 n+1}=$ $\mathbb{C}^{n+1}$ (biholomorphically). We assign to each $((a, w), \lambda \cdot A) \in \mathrm{A}(\mathcal{L})$ an element

$$
\left(\left[\begin{array}{c}
a \\
w
\end{array}\right],\left(\begin{array}{c|c}
\lambda^{2} & \lambda^{t} w J_{n} A \\
\hline 0 & \lambda A
\end{array}\right)\right) \in \operatorname{Aff}\left(\mathbb{C}^{2 n+1}\right) .
$$

Then the action (2.5) of $((a, w), \lambda \cdot A)$ on $\mathcal{L}$ coincides with the above affine transformation of $\mathbb{C}^{2 n+1}$. Moreover, it is easy to check that this correspondence is an injective homomorphism:

$$
\mathrm{A}(\mathcal{L}) \leq \mathrm{Aff}\left(\mathbb{C}^{2 n+1}\right) .
$$

As a consequence it follows

$$
\mathrm{A}(\mathcal{L}) \leq \operatorname{hol}(\mathcal{L}, \eta)
$$

Let $M$ be a smooth manifold. Suppose that there exists a maximal collection of charts $\left\{\left(U_{\alpha}, \varphi_{\alpha}\right)\right\}_{\alpha \in \Lambda}$ whose coordinate changes belong to $\mathrm{A}(\mathcal{L})$. More precisely, $M=\cup_{\alpha \in \Lambda} U_{\alpha}, \varphi_{\alpha}: U_{\alpha} \rightarrow \mathcal{L}$ is a diffeomorphism onto its image. If $U_{\alpha} \cap U_{\beta} \neq \emptyset$, then there exists a unique element $g_{\alpha \beta} \in \mathrm{A}(\mathcal{L})$ such that $g_{\alpha \beta}=\varphi_{\beta} \cdot \varphi_{\alpha}^{-1}$ on $\varphi_{\alpha}\left(U_{\alpha} \cap U_{\beta}\right)$. We say that $M$ is locally modelled on $(\mathrm{A}(\mathcal{L}), \mathcal{L})$ (Compare Kulkarni, 1978).

Here is a sufficient condition for the existence on complex contact structure.

Proposition 2.2 If a $(4 n+2)$-dimensional smooth manifold $M$ is locally modelled on $(\mathrm{A}(\mathcal{L}), \mathcal{L})$, then $M$ is a complex contact manifold. Moreover, $M$ is also a complex affinely flat manifold. 
Proof. First of all, we define a complex structure on $M$. Let $J_{0}$ be the standard complex structure on $\mathcal{L}=\mathbb{C}^{2 n+1}$. Define a complex structure $J_{\alpha}$ on $U_{\alpha}$ by setting $\varphi_{\alpha *} J_{\alpha}=J_{0} \varphi_{\alpha *}$ on $U_{\alpha}$ for each $\alpha \in \Lambda$. When $g_{\alpha \beta} \in \mathrm{A}(\mathcal{L})$, note that $g_{\alpha \beta *} J_{0}=J_{0} g_{\alpha \beta *}$ from (2.8). On $U_{\alpha} \cap U_{\beta}$, a calculation shows that $\varphi_{\beta *} J_{\alpha}=g_{\alpha \beta *} \varphi_{\alpha *} J_{\alpha}=J_{0} \varphi_{\beta *}$. Since $\varphi_{\beta *} J_{\beta}=J_{0} \varphi_{\beta *}$ by the definition, it follows $J_{\alpha}=J_{\beta}$ on $U_{\alpha} \cap U_{\beta}$. This defines a complex structure $J$ on $M$. In particular, each $\varphi_{\alpha}$ : $\left(U_{\alpha}, J\right) \rightarrow\left(\mathcal{L}, J_{0}\right)\left(=\mathbb{C}^{2 n+1}\right)$ is a holomorphic embedding. Let $\eta$ be the holomorphic 1-form on $\mathcal{L}$ as before. Define a family of local holomorphic 1 -forms $\left\{\omega_{\alpha}, U_{\alpha}\right\}_{\alpha \in \Lambda}$ by

$$
\omega_{\alpha}=\varphi_{\alpha}^{*} \eta \text { on } U_{\alpha}
$$

If $U_{\alpha} \cap U_{\beta} \neq \emptyset$, then there exists a unique element $g_{\alpha \beta} \in \mathrm{A}(\mathcal{L})$ such that $g_{\alpha \beta}=\varphi_{\beta} \cdot \varphi_{\alpha}^{-1}$. From $(2.6), g_{\alpha \beta}^{*} \eta=\mu_{\alpha \beta}^{2} \cdot \eta$ for some $\mu_{\alpha \beta} \in \mathbb{C}^{*}$. It follows $\omega_{\beta}=\mu_{\alpha \beta}^{2} \cdot \omega_{\alpha}$. Thus the family $\left\{\omega_{\alpha}, U_{\alpha}\right\}_{\alpha \in \Lambda}$ is a complex contact structure on $(M, J)$. Apart from the complex contact structure, since $\mathrm{A}(\mathcal{L}) \leq \mathrm{Aff}\left(\mathbb{C}^{2 n+1}\right)$ from $(2.8), M$ is also modelled on $\left(\mathrm{Aff}\left(\mathbb{C}^{2 n+1}\right)\right.$, $\mathbb{C}^{2 n+1}$ ) where $\mathcal{L}=\mathbb{C}^{2 n+1} . M$ is a complex affinely flat manifold.

Remark 2.3 (1) When a subgroup $\Gamma \leq \mathrm{A}(\mathcal{L})$ acts properly discontinuously and freely on a domain $\Omega$ of $\mathcal{L}$ with compact quotient, we obtain a compact complex contact manifold $\Omega / \Gamma$ by this proposition. In fact let $p: \Omega \rightarrow \Omega / \Gamma$ be a covering holomorphic projection. Take a set of evenly covered neighborhoods $\left\{U_{\alpha}\right\}_{\alpha \in \Lambda}$ of $\Omega / \Gamma$. Choose a family of open subsets $\tilde{U}_{\alpha}$ such that $p_{\alpha}=p_{\mid \tilde{U}_{\alpha}}: \tilde{U}_{\alpha} \rightarrow U_{\alpha}$ is a biholomorphism. Put $\omega_{\alpha}=\left(p_{\alpha}^{-1}\right)^{*} \eta$. Then the family $\left\{U_{\alpha}, \omega_{\alpha}\right\}_{\alpha \in \Lambda}$ is a complex contact structure on $\Omega / \Gamma$.

(2) When $\Omega=\mathcal{L}, \mathcal{L} / \Gamma$ is said to be a compact complete affinely flat manifold. Concerning the Auslandr-Milnor conjecture, we do not know whether the fundamental group $\Gamma$ is virtually polycyclic.

(3) By the monodromy argument, there exists a developing immersion: dev: $\tilde{M} \rightarrow \mathcal{L}$ from the universal covering $\tilde{M}$ of $M$. Then note that $\operatorname{dev}_{*} J=J_{0} \operatorname{dev}_{*}$, i.e. dev is a holomorphic map. Here $J$ is the lift of complex structure on $\tilde{M}$ (We wrote the same $J$ on $\tilde{M})$.

When $M$ is a complex manifold, we assume that the complex structure on $M$ coincides with the one constructed in Proposition 2.2.

\subsection{Complex Contact Similarity Geometry}

It is in general difficult to find such a properly discontinuous group $\Gamma$ as in Remark 2.3. $\mathrm{Sp}(n, \mathbb{C})$ contains a maximal compact symplectic subgroup $\operatorname{Sp}(n)=\left\{\left.A \in \mathrm{U}(2 n)\right|^{t} A \mathrm{~J}_{n} A=\mathrm{J}_{n}\right\}$ where $\operatorname{Sp}(n, \mathbb{C}) \cong \operatorname{Sp}(n) \times \mathbb{R}^{n(2 n+1)}$.

Definition 2.4 Put $\operatorname{Sim}(\mathcal{L})=\mathcal{L} \rtimes\left(\operatorname{Sp}(n) \cdot \mathbb{C}^{*}\right) \leq \mathrm{A}(\mathcal{L})$. The pair $(\operatorname{Sim}(\mathcal{L}), \mathcal{L})$ is called complex contact similarity geometry. If a manifold $M$ is locally modelled on this geometry, $M$ is said to be a complex contact similarity manifold. The euclidean subgroup of $\operatorname{Sim}(\mathcal{L})$ is defined to be $\mathrm{E}(\mathcal{L})=\mathcal{L} \rtimes\left(\operatorname{Sp}(n) \cdot S^{1}\right)$.

For example, choose $c \in \mathbb{C}^{*}$ with $|c| \neq 1$ and $A \in \operatorname{Sp}(n)$. Put $r=((0,0), c \cdot A) \in \operatorname{Sim}(\mathcal{L})$. Let $\mathbb{Z}^{+}$be an infinite cyclic group generated by $r$. Then it is easy to see that $\mathbb{Z}^{+}$acts freely and properly discontinuously on the complement $\mathcal{L}-\{0\}$. Here $0=(0,0) \in \mathcal{L}_{2 n+1}=\mathcal{L}$. The quotient $\mathcal{L}-\{0\} / \mathbb{Z}^{+}$is diffeomorphic to $S^{1} \times S^{4 n+1}$. By Proposition 2.2 ((1) of Remark 2.3), $S^{1} \times S^{4 n+1}$ is a complex contact similarity manifold.

Let $\mathbb{H}^{n}$ be the $4 n$-dimensional quaternionic vector space. The quaternionic similarity group $\operatorname{Sim}\left(\mathbb{H}^{n}\right)=\mathbb{H}^{n} \rtimes$ $\left((\operatorname{Sp}(n) \cdot \operatorname{Sp}(1)) \times \mathbb{R}^{+}\right)\left(\right.$resp. quaternionic euclidean group $\left.\mathrm{E}\left(\mathbb{H}^{n}\right)=\mathbb{H}^{n} \rtimes(\operatorname{Sp}(n) \cdot \operatorname{Sp}(1))\right)$ has a special subgroup $\widehat{\operatorname{Sim}}\left(\mathbb{H}^{n}\right)=\mathbb{H}^{n} \rtimes\left(\left(\operatorname{Sp}(n) \cdot S^{1}\right) \times \mathbb{R}^{+}\right)\left(\right.$resp. $\left.\widehat{\mathrm{E}}\left(\mathbb{H}^{n}\right)=\mathbb{H}^{n} \rtimes\left(\operatorname{Sp}(n) \cdot S^{1}\right)\right)$. When we identify $\mathbb{H}^{n}$ with the complex vector space $\mathbb{C}^{2 n}$ by the correspondence $(a+b \mathbf{j}) \mapsto(\bar{a}, b), \overline{\operatorname{Sim}}\left(\mathbb{H}^{n}\right)$ is canonically isomorphic to the complex similarity subgroup $\mathbb{C}^{2 n} \rtimes\left(\operatorname{Sp}(n) \cdot \mathbb{C}^{*}\right)$ where $\mathbb{C}^{*}=S^{1} \times \mathbb{R}^{+}$. Then there are commutative exact sequences:

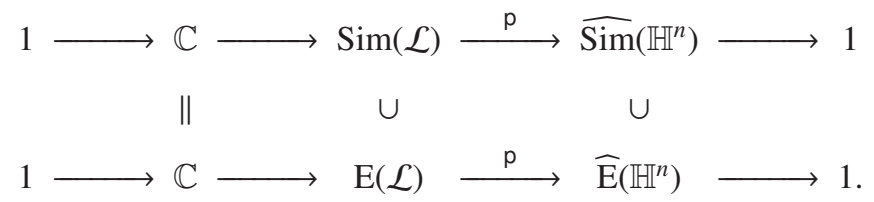

Choosing a torsionfree discrete cocompact subgroup $\Gamma$ from $\mathrm{E}(\mathcal{L})$, we obtain an infranilmanifold $\mathcal{L} / \Gamma$ of complex dimension $2 n+1$. In particular, $\Gamma \cap \mathcal{L}$ is discrete uniform in $\mathcal{L}$ by the Auslander-Bieberbach theorem. As $\mathbb{C}$ is the central subgroup of $\mathcal{L}, \Gamma \cap \mathbb{C}$ is discrete uniform in $\mathbb{C}$ and so $\Delta=\mathrm{p}(\Gamma)$ is a discrete uniform subgroup in $\widehat{\mathrm{E}}\left(\mathbb{H}^{n}\right)$. We obtain a Seifert singular fibration over a quaternionic euclidean orbifold $\mathbb{H}^{n} / \Delta: T_{\mathbb{C}}^{1} \rightarrow \mathcal{L} / \Gamma \longrightarrow \mathbb{H}^{n} / \Delta$. By (1) of Remark $2.3, \mathcal{L} / \Gamma$ is a complex contact manifold. 
Remark 2.5 When we take a finite index nilpotent subgroup $\Gamma^{\prime}$ of $\Gamma$ admitting a central extension: $1 \rightarrow \mathbb{Z}^{2} \rightarrow$ $\Gamma^{\prime} \longrightarrow \mathbb{Z}^{4 n} \rightarrow 1$, a nilmanifold $\mathcal{L} / \Gamma^{\prime}$ admits a holomorphic principal $T_{\mathbb{C}}^{1}$-bundle over a complex torus $T_{\mathbb{C}}^{2 n}=$ $\mathbb{H}^{n} / \mathbb{Z}^{4 n}$. This holomorphic example is a special case of Foreman's $T^{2}$ - connection bundle over $T_{\mathbb{C}}^{2 n}$ (Foreman, 2000).

We give rise to a classification of compact complex contact similarity manifolds under the existence of $S^{1}$-actions (Compare Fried, 1980; Miner, 1991 for the related results of similarity manifolds). Recall that $\operatorname{Aut}_{c c}(M)$ is the group of complex contact transformations from Definition 2.1.

Theorem 2.6 Let $M$ be a $4 n+2$-dimensional compact complex contact similarity manifold. If $S^{1} \leq \mathrm{Aut}_{c c}(M)$ acts on $M$ without fixed points, then $M$ is holomorphically diffeomorphic to a complex contact infranilmanifold $\mathcal{L} / \Gamma$ or a complex contact Hopf manifold $S^{1} \times S^{4 n+1}$.

Proof. Let $J$ be a complex structure on $M$. Given a collection of charts $\left\{U_{\alpha}, \varphi_{\alpha}, J_{\alpha}\right\}$ on $M$ with $J_{\alpha}=J_{\mid U_{\alpha}}$ such that $\varphi_{\alpha}:\left(U_{\alpha}, J_{\alpha}\right) \rightarrow\left(\mathcal{L}, J_{0}\right)$ is a holomorphic diffeomorphism onto its image, the monodromy argument shows that there is a developing pair:

$$
(\rho, \operatorname{dev}):\left(\operatorname{Aut}_{c c}(\tilde{M}), \tilde{M}\right) \rightarrow(\operatorname{Sim}(\mathcal{L}), \mathcal{L})
$$

where $\tilde{M}$ is the universal covering and $\tilde{J}$ is a lift of $J$ to $\tilde{M}$, and $\pi=\pi_{1}(M) \leq \operatorname{Aut}_{c c}(\tilde{M})$. Then dev is a holomorphic immersion $\operatorname{dev}_{*} J=J_{0} \operatorname{dev}_{*}$ and $\rho$ : $\operatorname{Aut}_{c c}(\tilde{M}) \rightarrow \operatorname{Sim}(\mathcal{L})$ is a holonomony homomorphism. Put $\Gamma=\rho(\pi)$. Let $\tilde{S}^{1}$ be a lift of $S^{1}$ to $\tilde{M}$ so that $\rho\left(\tilde{S}^{1}\right) \leq \operatorname{Sim}(\mathcal{L})$.

Case 1) If $\Gamma \leq \mathrm{E}(\mathcal{L})$, then there is a $\mathrm{E}(\mathcal{L})$-invariant Riemannian metric on $\mathcal{L}$. As $M$ is compact, the pullback metric on $\tilde{M}$ by dev is (geodesically) complete, dev: $\tilde{M} \rightarrow \mathcal{L}$ is an isometry. As dev becomes a complex contact diffemorphism, $M$ is holomorphically isomorphic to a complex contact infranilmanifold $\mathcal{L} / \Gamma$.

Case 2) Suppose that some $\rho(\gamma)$ has a nontrivial summand in $\mathbb{R}^{+} \leq \mathcal{L} \rtimes\left(\operatorname{Sp}(n) \cdot S^{1} \times \mathbb{R}^{+}\right)=\operatorname{Sim}(\mathcal{L})$. In view of the affine representation $\rho(\gamma)=(p, P)$ where $P=\left(\begin{array}{c|c}\lambda^{2} & \lambda^{t} w J_{n} A \\ \hline 0 & \lambda A\end{array}\right)$ from (2.7), we note $|\lambda| \neq 1$, i.e. $P$ has no eigenvalue 1. Then there exists an element $z_{0} \in \mathcal{L}$ such that the conjugate $\left(z_{0}, I\right) \rho(\gamma)\left(-z_{0}, I\right)=(0, P)$. We may assume that $\rho(\gamma)=(0, P) \in \operatorname{Aff}(\mathcal{L})$ from the beginning. As $\rho\left(\tilde{S}^{1}\right)$ centralizes $\Gamma$, if $\rho(t)=(q, Q) \in \rho\left(\tilde{S}^{1}\right)$, then the equation $\rho(t) \rho(\gamma)=\rho(\gamma) \rho(t)$ implies that $P q=q$ and so $q=0$. Thus $\rho(t)=(0, Q)=\left((0,0), \mu_{t} \cdot B_{t}\right) \in \operatorname{Sp}(n) \cdot S^{1} \times \mathbb{R}^{+} \leq \operatorname{Sim}(\mathcal{L})$. It follows $\rho\left(\tilde{S}^{1}\right) \leq \operatorname{Sp}(n) \cdot S^{1} \times \mathbb{R}^{+}$. In particular, $\rho\left(\tilde{S}^{1}\right)$ has a non-empty fixed point set $\mathcal{S}$ in $\mathcal{L}$. If $\operatorname{dev}(x) \in \mathcal{S}$, then $\operatorname{dev}\left(\tilde{S}^{1} x\right)=\rho\left(\tilde{S}^{1}\right) \operatorname{dev}(x)=x$. Since dev is an immersion, $\tilde{S}^{1} x=x$. As $S^{1}$ has no fixed points on $M$, it is noted that $\operatorname{dev}(\tilde{M}) \subset \mathcal{L}-\mathcal{S}$. Let $\operatorname{Sim}(\mathcal{L}-\mathcal{S})$ be the subgroup of $\operatorname{Sim}(\mathcal{L})$ whose elements leave $\mathcal{S}$ invariant. Note that $\Gamma \leq \operatorname{Sim}(\mathcal{L}-\mathcal{S})$.

We determine $\mathcal{S}$ and $\operatorname{Sim}(\mathcal{L}-\mathcal{S})$. Since $\rho\left(\tilde{S}^{1}\right)$ belongs to the maximal abelian group $T^{2 n} \cdot S^{1} \times \mathbb{R}^{+}$up to conjugate in $\operatorname{Sp}(n) \cdot S^{1} \times \mathbb{R}^{+}$, we can put $\left\langle\lambda_{t}\right\rangle \leq S^{1} \times \mathbb{R}^{+},\left\langle s_{t}\right\rangle=S^{1}$ and

$$
\begin{aligned}
& \left.\rho\left(\tilde{S}^{1}\right)=\left\{\left((0,0), \mu_{t} \cdot B_{t}\right)\right)=\left(\left[\begin{array}{l}
0 \\
0
\end{array}\right],\left(\begin{array}{c|c}
\mu_{t}^{2} & 0 \\
\hline 0 & \mu_{t} B_{t}
\end{array}\right)\right)\right\} \\
& B_{t}=\left(\begin{array}{cccccc}
s_{t} & & & & & \\
& \ddots & & & & \\
& & s_{t} & & & \\
& & & 1 & & \\
& & & & \ddots & \\
& & & & & 1
\end{array}\right) \in T^{2 n} \leq \operatorname{Sp}(n)
\end{aligned}
$$

where $\mathrm{Sp}(n) \leq \mathrm{U}(2 n)$ is canonically embedded so that $2 k$-numbers of $s_{t}$ 's and $2 \ell$-numbers of 1 's. Recall that $\rho\left(\tilde{S}^{1}\right)$ acts on $\mathcal{L}$ by $\rho(t)\left(z_{0}, z\right)=\left(\mu_{t}^{2} z_{0}, \mu_{t} B_{t} z\right)$.

Case I. $\mu_{t} \neq 1$. Suppose that $\mu_{t} \lambda_{t}=1$. Then $\mathcal{S}=\operatorname{Fix}\left(\rho\left(\tilde{S}^{1}\right), \mathcal{L}\right)=\left\{(0,(z, 0)) \in \mathcal{L} \mid z \in \mathbb{C}^{2 k}\right\}(0 \leq k \leq n)$. As the element $((a, w), \lambda \cdot A) \in \operatorname{Sim}(\mathcal{L})$ acts by $((a, w), \lambda \cdot A)(0,(z, 0))=\left(a+\lambda^{t} w J_{n} A z, w+\lambda A z\right) \in \mathcal{S}$ (cf. (2.5)), we can check that $a=0, w \in \mathbb{C}^{2 k}$ and so $\lambda A z \in \mathbb{C}^{2 k}$. In particular, $A \in \mathrm{Sp}(k)$. From $w \mathrm{~J}_{n} A z=0$, it follows $w=0$.

$$
\operatorname{Sim}(\mathcal{L}-\mathcal{S})=\{((0,0), \lambda \cdot A) \mid A \in \operatorname{Sp}(k)\}=\operatorname{Sp}(k) \cdot S^{1} \times \mathbb{R}^{+} .
$$


Case II. $\mu_{t}=1$. Then $\mathcal{S}=\left\{\left(z_{0},(0, z)\right) \in \mathcal{L} \mid z \in \mathbb{C}^{2 \ell}\right\}=\mathcal{L}_{2 \ell+1}(0 \leq \ell \leq n-1)$. It follows as above

$$
\begin{aligned}
\operatorname{Sim}(\mathcal{L}-\mathcal{S}) & =\left\{((a, w), \lambda \cdot A) \mid w \in \mathbb{C}^{2 \ell}, A \in \operatorname{Sp}(\ell)\right\} \\
& =\mathcal{L}_{2 \ell+1} \rtimes\left(\operatorname{Sp}(\ell) \cdot S^{1} \times \mathbb{R}^{+}\right)=\operatorname{Sim}\left(\mathcal{L}_{2 \ell+1}\right) .
\end{aligned}
$$

We need the following lemma.

Lemma 2.7 $\operatorname{Sim}(\mathcal{L}-\mathcal{S})$ acts properly on $\mathcal{L}-\mathcal{S}$.

Proof. Case I. There is an equivariant inclusion

$$
\left(\operatorname{Sp}(k) \cdot S^{1} \times \mathbb{R}^{+}, \mathcal{L}-\mathcal{S}\right) \subset\left(\operatorname{Sp}(n) \cdot S^{1} \times \mathbb{R}^{+}, \mathcal{L}-\{0\}\right) .
$$

As there is an $\operatorname{Sp}(n) \cdot S^{1} \times \mathbb{R}^{+}$-invariant Riemannian metric on $\mathcal{L}-\{0\}$ and $\operatorname{Sp}(k) \cdot S^{1} \times \mathbb{R}^{+}$is a closed subgroup, it acts properly on $\mathcal{L}-\mathcal{S}$.

Case II. Let $G=\mathbb{C}^{2 \ell} \rtimes\left(\operatorname{Sp}(\ell) \cdot S^{1} \times \mathbb{R}^{+}\right)$be the semidirect group which preserves the complement $\mathbb{C}^{2 n}-\mathbb{C}^{2 \ell}$. Then there is an equivariant principal bundle:

$$
(\mathbb{C}, \mathbb{C}) \rightarrow\left(\operatorname{Sim}\left(\mathcal{L}_{2 \ell+1}\right), \mathcal{L}-\mathcal{L}_{2 \ell+1}\right) \longrightarrow\left(G, \mathbb{C}^{2 n}-\mathbb{C}^{2 \ell}\right) .
$$

We note that $G$ acts properly on $\mathbb{C}^{2 n}-\mathbb{C}^{2 \ell}$. For this, we observe that

$$
\mathbb{C}^{2 n}-\mathbb{C}^{2 \ell}=S^{4 n}-S^{4 \ell}=\mathbb{H}_{\mathbb{R}}^{4 \ell+1} \times S^{4 n-4 \ell-1}
$$

in which

$$
G \leq \mathbb{R}^{4 \ell} \rtimes\left(\mathrm{O}(4 \ell) \times \mathbb{R}^{+}\right)=\operatorname{Sim}\left(\mathbb{R}^{4 \ell}\right) \leq \mathrm{PO}(4 \ell+1,1) .
$$

As $\mathrm{PO}(4 \ell+1,1) \times \mathrm{O}(4 n-4 \ell)=\operatorname{Isom}\left(\mathbb{H}_{\mathbb{R}}^{4 \ell+1} \times S^{4 n-4 \ell-1}\right)$ and $G$ is a closed subgroup of $\mathrm{PO}(4 \ell+1,1), G$ acts properly on $\mathbb{C}^{2 n}-\mathbb{C}^{2 \ell}$.

Since $\mathbb{C}$ acts properly on $\mathcal{L}-\mathcal{L}_{2 \ell+1}$, the above principal bundle (2.16) implies that $\operatorname{Sim}\left(\mathcal{L}_{2 \ell+1}\right)$ acts properly on $\mathcal{L}-\mathcal{L}_{2 \ell+1}$.

We continue the proof of Theorem 2.6. For Case I, there is an $\operatorname{Sp}(k) \cdot S^{1} \times \mathbb{R}^{+}$-invariant Riemannian metric on $\mathcal{L}-\mathcal{S}$. Put $H=\operatorname{Sp}(k) \cdot S^{1} \times \mathbb{R}^{+}$. As $\mathcal{L}-\mathcal{S}=\mathbb{C}^{2 n+1}-\mathbb{C}^{2 k}=\mathbb{H}_{\mathbb{R}}^{4 k+1} \times S^{4 n-4 k+1}$ where $H \leq \operatorname{Sim}\left(\mathbb{R}^{4 k}\right) \leq \mathrm{PO}(4 k+1,1)$, note that the quotient $\mathcal{L}-\mathcal{S} / H$ is a Hausdorff space. On the other hand,

$$
\begin{aligned}
\mathcal{L}-\mathcal{S} / H & =\mathbb{H}_{\mathbb{R}}^{4 k+1} / H \times S^{4 n-4 k+1} \\
& =\mathbb{R}^{4 k} \rtimes \mathbb{R}^{+} / H \times S^{4 n-4 k+1} \\
& =\mathbb{R}^{4 k} /\left(\operatorname{Sp}(k) \cdot S^{1}\right) \times S^{4 n-4 k+1} .
\end{aligned}
$$

$\mathcal{L}-\mathcal{S} / H$ cannot be compact unless $k=0$.

On the other hand, as $M$ is compact and $\Gamma \leq \operatorname{Sim}(\mathcal{L}-\mathcal{S})$, using Lemma 2.7, dev : $\tilde{M} \rightarrow \mathcal{L}-\mathcal{S}$ is a covering map. $\mathcal{L}-\mathcal{S}$ is simply connected unless $k=n$. Then $M \cong \mathcal{L}-\mathcal{S} / \Gamma$ is compact $(k \neq n)$. If we consider the fiber space $\mathcal{L}-\mathcal{S} / \Gamma \rightarrow \mathcal{L}-\mathcal{S} / H, \mathcal{L}-\mathcal{S} / H$ must be compact, which cannot occur except for $k=0$.

If $\mathcal{L}-\mathcal{S}=\mathbb{H}_{\mathbb{R}}^{4 n+1} \times S^{1}(k=n)$, then there is a lift of dev, $\widetilde{\operatorname{dev}}: \tilde{M} \rightarrow \mathbb{H}_{\mathbb{R}}^{4 n+1} \times \mathbb{R}$ which is a diffeomorphism. The group $\tilde{\Gamma}=\widetilde{\operatorname{dev}} \circ \pi \circ \widetilde{\operatorname{dev}}^{-1}$ acts properly discontinuously and freely on $\mathbb{H}_{\mathbb{R}}^{4 n+1} \times \mathbb{R}$ such that $\mathbb{H}_{\mathbb{R}}^{4 n+1} \times \mathbb{R} / \tilde{\Gamma}$ is compact. As there is the canonical projection:

$$
\mathbb{H}_{\mathbb{R}}^{4 n+1} \times \mathbb{R} / \tilde{\Gamma} \rightarrow \mathbb{H}_{\mathbb{R}}^{4 n+1} \times S^{1} / H,
$$

$\mathbb{H}_{\mathbb{R}}^{4 n+1} \times S^{1} / H$ is compact. This case is also impossible.

For $k=0, \mathcal{S}=\{0\}$, dev: $\tilde{M} \rightarrow \mathcal{L}-\{0\}$ is a diffeomorphism. As $\Gamma \leq S^{1} \times \mathbb{R}^{+}$acting freely on $\mathcal{L}-\{0\}=\mathbb{H}_{\mathbb{R}}^{1} \times S^{4 n+1}$, $M$ is biholomorphic to $\mathcal{L}-\{0\} / \Gamma$ which is diffeomorphic with $S^{1} \times S^{4 n+1}$.

For Case II, $\mathcal{L}-\mathcal{L}_{2 \ell+1}$ is always simply connected (cf. (2.16)). Then $M$ is diffeomorphic to $\mathcal{L}-\mathcal{L}_{2 \ell+1} / \Gamma$ so that $\Gamma \leq \operatorname{Sim}\left(\mathcal{L}_{2 \ell+1}\right)=\mathcal{L}_{2 \ell+1} \rtimes\left(\operatorname{Sp}(\ell) \cdot S^{1} \times \mathbb{R}^{+}\right)$is a discrete subgroup. As there is a fiber space

$$
\operatorname{Sim}\left(\mathcal{L}_{2 \ell+1}\right) / \Gamma \rightarrow \mathcal{L}-\mathcal{L}_{2 \ell+1} / \Gamma \longrightarrow \mathcal{L}-\mathcal{L}_{2 \ell+1} / \operatorname{Sim}\left(\mathcal{L}_{2 \ell+1}\right),
$$


it follows that $\operatorname{Sim}\left(\mathcal{L}_{2 \ell+1}\right) / \Gamma$ is compact. Since $\mathcal{L}_{2 \ell+1}$ is a maximal nilpotent subgroup of $\operatorname{Sim}\left(\mathcal{L}_{2 \ell+1}\right), \mathcal{L}_{2 \ell+1} \cap \Gamma$ is discrete uniform in $\mathcal{L}_{2 \ell+1}$. As $\mathbb{R}^{+}$acts on $\mathcal{L}$ as multiplication, $\Gamma$ cannot have a nontrivial summand in $\mathbb{R}^{+}$. This contradicts the hypothesis of Case 2. So Case II does not occur. This proves the theorem.

\section{Connected Sum}

In Kobayashi (1959), there is a complex contact structure on the complex projective space $\mathbb{C P}^{2 n+1}$; let $\omega=$ $\sum_{i=1}^{n+1}\left(z_{2 i-1} \cdot d z_{2 i}-z_{2 i} \cdot d z_{2 i-1}\right)$ be a holomorphic 1 -form on $\mathbb{C}^{2 n+2}$. Put $U_{i}=\left\{\left[w_{0}, \ldots, w_{2 n+1}\right] \mid w_{i} \neq 0\right\}$ which forms a cover $\left\{U_{i}\right\}$ of $\mathbb{C P}^{2 n+1}$. If $s_{i}$ is a holomorphic cross-section of the principal bundle $\mathbb{C}^{*} \rightarrow \mathbb{C}^{2 n+2}-\{0\} \longrightarrow \mathbb{C P}^{2 n+1}$ restricted to $U_{i}$, setting $\omega_{i}=s_{i}^{*} \omega,\left\{\omega_{i}\right\}$ defines a complex contact structure on $\mathbb{C P}^{2 n+1}$. For example, let $\iota: U_{0} \rightarrow \mathbb{C}^{2 n+1}$ be the local coordinate system defined by $\iota\left(\left[w_{0}, \ldots, w_{2 n+1}\right]\right)=\left(z_{0}, \ldots, z_{2 n}\right)$ such that $w_{i+1} / w_{0}=z_{i}$. A holomorphic map $s_{0}: U_{0} \rightarrow \mathbb{C}^{2 n+2}-\{0\}$ may be defined as

$$
s_{0} \circ \iota^{-1}\left(z_{0}, \ldots, z_{2 n}\right)=\left(1, z_{0},-z_{1}, z_{2},-z_{3}, z_{4}, \ldots,-z_{2 n-1}, z_{2 n}\right) .
$$

Then the holomorphic 1-form $\left(s_{0} \circ \iota^{-1}\right)^{*} \omega$ on $\iota\left(U_{0}\right)$ is described as

$$
\left(s_{0} \circ \iota^{-1}\right)^{*} \omega=d z_{0}-\sum_{i=1}^{n}\left(z_{2 i-1} \cdot d z_{2 i}-z_{2 i} \cdot d z_{2 i-1}\right) .
$$

For this,

$$
\begin{aligned}
\left(s_{0} \circ \iota^{-1}\right)^{*} \omega & =\left(s_{0} \circ \iota^{-1}\right)^{*}\left(\left(z_{1} d z_{2}-z_{2} d z_{1}\right)+\left(z_{3} d z_{4}-z_{4} d z_{3}\right)+\cdots+\left(z_{2 n+1} d z_{2 n+2}-z_{2 n+2} d z_{2 n+1}\right)\right) \\
& =d z_{0}-\left(z_{1} d z_{2}-z_{2} d z_{1}\right)-\cdots-\left(z_{2 n-1} \cdot d z_{2 n}-z_{2 n} d z_{2 n-1}\right) .
\end{aligned}
$$

So $\left(s_{0} \circ \iota^{-1}\right)^{*} \omega$ is equivalent with $\eta_{\mid \iota\left(U_{0}\right)}$ of (2.2).

Let $p: \mathcal{L} \rightarrow \mathcal{L} / \Gamma$ be the holomorphic covering map. Put $V_{0}=p\left(\iota\left(U_{0}\right)\right)$ and $p(0)=x$. Then the map $p \circ \iota: U_{0} \rightarrow V_{0}$ is a holomorphic map with $p \circ \iota([1,0, \ldots, 0])=x$. Choose a neighborhood $U_{0}^{\prime} \subset U_{0}$ such that $\iota\left(U_{0}^{\prime}\right)$ is a closed ball $B$ at the origin in $\mathbb{C}^{2 n+1}$. Put $p(B)=V_{0}^{\prime} \subset V_{0}$ so that $p \circ \iota: U_{0}^{\prime} \rightarrow V_{0}^{\prime}$ is a biholomorphism. Then a connected sum $\mathbb{C} \mathbb{P}^{2 n+1} \# \mathcal{L} / \Gamma$ is obtained by glueing $\mathbb{C P}^{2 n+1}-\operatorname{int} U_{0}^{\prime}$ and $\mathcal{L} / \Gamma-\operatorname{int} V_{0}^{\prime}$ along the boundaries $\partial U_{0}^{\prime}$ and $\partial V_{0}^{\prime}$ by $p \circ \iota$.

Proposition 3.1 The connected sum $\mathbb{C P}^{2 n+1} \# \mathcal{L} / \Gamma$ admits a complex contact structure.

Proof. As above $\left(s_{0} \circ \iota^{-1}\right)^{*} \omega=\eta$ on $\iota\left(U_{0}\right)$. Note that $\omega_{0}=s_{0}^{*} \omega=\iota^{*} \eta$ on $U_{0}$. On the other hand, the complex contact structure $\left\{\eta_{i}\right\}$ on $\mathcal{L} / \Gamma$ satisfies that $p^{*} \eta_{0}=\eta$ on $\iota\left(U_{0}\right)$. The holomorphic map $p \circ \iota: U_{0} \rightarrow V_{0}$ satisfies that $(p \circ \iota)^{*} \eta_{0}=\omega_{0}$. Since $J(p \circ \iota)_{*}=(p \circ \iota)_{*} J$ on $U_{0}$, the complex structure $J$ is naturally extended to a complex structure on $\mathbb{C} \mathbb{P}^{2 n+1} \# \mathcal{L} / \Gamma$ along the boundary $\partial U_{0}^{\prime}$.

Since any complex contact similarity manifold $M$ is locally modelled on $(\operatorname{Sim}(\mathcal{L}), \mathcal{L})$ by the definition, every point of $M$ has a neighborhood $U$ on which the complex contact structure is equivalent to a restriction of $(\eta, \mathcal{L})$. Similarly to the above proof, we have

Theorem 3.2 Any connected sum $M_{1} \# \ldots \# M_{k} \# \ell \mathbb{C P}^{2 n+1}$ admits a complex contact structure for a finite number of complex contact similarity manifolds $M_{1}, \ldots, M_{k}$ and $\ell$-copies of $\mathbb{C P}^{2 n+1}$.

\section{Contact Complex Structure from Quaternionic Heisenberg Lie Group}

\subsection{Quaternionic Heisenberg Geometry}

Denote $\mathbb{R}^{3}=\operatorname{Im} \mathbb{H}$ which is the imaginary part of the quaternion field $\mathbb{H}$. $\mathcal{M}$ is the product $\mathbb{R}^{3} \times \mathbb{H}^{n}$ with group law:

$$
(\alpha, u) \cdot(\beta, v)=(\alpha+\beta+\operatorname{Im}\langle u, v\rangle, u+v)
$$

Here $\langle u, v\rangle={ }^{t} \bar{u} \cdot v=\sum_{i=1}^{n} \bar{u}_{i} v_{i}$ is the Hermitian inner product where $\bar{u}=\left(\bar{u}_{1}, \ldots, \bar{u}_{n}\right)$ is the quaternion conjugate. $\mathcal{M}$ is nilpotent because $[\mathcal{M}, \mathcal{M}]=\mathbb{R}^{3}$ which is the center consisting of the form $((a, b, c), 0)(a, b, c \in \mathbb{R})$. $\mathcal{M}$ is called quaternionic Heisenberg Lie group. The similarity subgroup $\operatorname{Sim}(\mathcal{M})$ is defined to be the semidirect product $\mathcal{M} \rtimes\left(\operatorname{Sp}(n) \cdot \operatorname{Sp}(1) \times \mathbb{R}^{+}\right)$. The action of $\operatorname{Sim}(\mathcal{M})$ on $\mathcal{M}$ is given as follows; for $h=((\alpha, u),(A \cdot g, t)) \in$ $\mathcal{M} \rtimes\left(\operatorname{Sp}(n) \cdot \operatorname{Sp}(1) \times \mathbb{R}^{+}\right),(\beta, v) \in \mathcal{M}$,

$$
h \circ(\beta, v)=\left(\alpha+t^{2} g \beta g^{-1}+\operatorname{Im}\left\langle u, t A v g^{-1}\right\rangle, u+t \cdot A v g^{-1}\right) .
$$


The pair $(\operatorname{Sim}(\mathcal{M}), \mathcal{M})$ is called quaternionic Heisenberg geometry.

Let $u_{i}=z_{i}+w_{i} \mathbf{j} \in \mathbb{H}\left(z_{i}, w_{i} \in \mathbb{C}\right)$. It is easy to check that the correspondence $\mathrm{p}: \mathcal{M} \rightarrow \mathcal{L}$ defined by

$$
\left(a \mathbf{i}+b \mathbf{j}+c \mathbf{k},\left(u_{1}, \ldots, u_{n}\right)\right) \mapsto\left(b+c \mathbf{i},\left(\bar{z}_{1}, w_{1}, \bar{z}_{2}, w_{2}, \ldots, \bar{z}_{n}, w_{n}\right)\right)
$$

is a Lie group homomorphism. Let $\widehat{\operatorname{Sim}}(\mathcal{M})=\mathcal{M} \rtimes\left(\operatorname{Sp}(n) \cdot S^{1} \times \mathbb{R}^{+}\right)$be the subgroup of $\operatorname{Sim}(\mathcal{M})$. Then $\mathrm{p}: \mathcal{M} \rightarrow \mathcal{L}$ induces a homomorphism q: $\widehat{\operatorname{Sim}}(\mathcal{M}) \rightarrow \operatorname{Sim}(\mathcal{L})$ for which $(\mathrm{q}, \mathrm{p}):(\widehat{\operatorname{Sim}}(\mathcal{M}), \mathcal{M}) \rightarrow(\operatorname{Sim}(\mathcal{L}), \mathcal{L})$ is equivariant.

Take the coordinates $(a, b, c) \in \mathbb{R}^{3}, u=\left(u_{1}, \ldots, u_{n}\right) \in \mathbb{H}^{n}$. Define a $\operatorname{Im} \mathbb{H}$-valued 1 -form on $\mathcal{M}$ to be

$$
\omega=d a \mathbf{i}+d b \mathbf{j}+d c \mathbf{k}-\operatorname{Im}\langle u, d u\rangle .
$$

We may put

$$
\omega=\omega_{1} \mathbf{i}+\omega_{2} \mathbf{j}+\omega_{3} \mathbf{k}
$$

for some real 1-forms $\omega_{1}, \omega_{2}, \omega_{3}$ on $\mathcal{M}$. Noting (4.1), $p^{*} \eta \cdot \mathrm{j}$ is a $\mathbb{C j}(\leq \mathbb{H})$-valued 1-form on $\mathcal{M}$. A calculation shows that

$$
\omega-\mathrm{p}^{*} \eta \cdot \mathrm{j}=d a \mathrm{i}+\sum_{i=1}^{n}\left(\bar{z}_{i} d z_{i}-z_{i} d \bar{z}_{i}+w_{i} d \bar{w}_{i}-\bar{w}_{i} d w_{i}\right)
$$

which is an $\mathbb{R i}$-valued 1 -form. Then we have from (4.3) that

$$
\omega-\mathrm{p}^{*} \eta \cdot \mathrm{j}=\omega_{1} \cdot \mathrm{i} .
$$

In particular when $\mathrm{p}_{*}: T \mathcal{M} \rightarrow T \mathcal{L}$ is the differential map, this equality shows

$$
\mathrm{p}_{*}(\operatorname{Ker} \omega)=\operatorname{Ker} \eta \text {. }
$$

\subsection{Quaternionic Carnot-Carathéodory Structure on $\mathcal{M}^{4 n+3}$}

Let $v: \mathcal{M} \rightarrow \mathbb{H}^{n}$ be the projection defined by $v((a, b, c), u)=u$. Then it is easy to check that $v_{*}: \operatorname{Ker} \omega \rightarrow T \mathbb{H}^{n}$ is an isomorphism at each point. By the pullback of this isomorphism, the standard quaternionic structure $\left\{J_{1}, J_{2}, J_{3}\right\}$ on $\mathbb{H}^{n}$ induces an almost quaternionic structure on $\operatorname{Ker} \omega$. (We write it as $\left\{J_{1}, J_{2}, J_{3}\right\}$ also.) As $[\operatorname{Ker} \omega, \operatorname{Ker} \omega]=$ $\mathbb{R}^{3},\left(\operatorname{Ker} \omega,\left\{J_{\alpha}\right\}_{\alpha=1,2,3}\right)$ is said to be quaternionic Carnot-Carathéodory structure on $\mathcal{M}^{4 n+3}$ (cf. Alekseevsky \& Kamishima, 2008).

Set $u_{i}=z_{i}+w_{i} \mathbf{j}=x_{i}+y_{i} \mathbf{i}+\left(p_{i}+q_{i} \mathbf{i}\right) \mathbf{j}$, so that

$$
g=|d u|^{2}=\sum_{i=1}^{n}\left(d x_{i}^{2}+d y_{i}^{2}+d p_{i}^{2}+d q_{i}^{2}\right)
$$

is the standard positive definite symmetric bilinear form on $\operatorname{Ker} \omega$. Since $d \omega=-d \bar{u} \wedge d u=d \omega_{1} \mathbf{i}+d \omega_{2} \mathbf{j}+d \omega_{3} \mathbf{k}$ from (4.2), (4.3), a reciprocity of the quaternionic structure shows that

$$
d \omega_{1}\left(J_{1} X, Y\right)=d \omega_{2}\left(J_{2} X, Y\right)=d \omega_{3}\left(J_{3} X, Y\right)=-g(X, Y) . \quad(\forall X, Y \in \operatorname{Ker} \omega) .
$$

Let $J_{0}$ be the complex structure on $\mathcal{L}$ and $\mu: \mathcal{L} \rightarrow \mathbb{C}^{2 n}$ the canonical projection. Since $\eta$ is a holomorphic 1form, $\mu_{*}:\left(\operatorname{Ker} \eta, J_{0}\right) \rightarrow\left(T \mathbb{C}^{2 n}, J_{0}\right)$ is an equivariant isomorphism. If q: $\mathbb{H}^{n} \rightarrow \mathbb{C}^{2 n}$ is an isomorphism defined by $\mathrm{q}\left(u_{1}, \ldots, u_{n}\right)=\left(\bar{z}_{1}, w_{1}, \ldots, \bar{z}_{n}, w_{n}\right)$, then there is the commutative diagram:

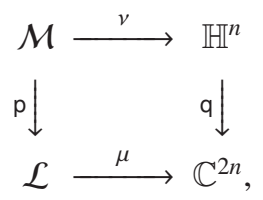

By the definition of $J_{1}, \mathrm{q}_{*} \circ J_{1}=J_{0} \circ \mathrm{q}_{*}$ on $T \mathbb{H}^{n}$.

Note that $\operatorname{Ker} \omega_{1}=\operatorname{Ker} \omega \oplus\left\langle\frac{d}{d b}, \frac{d}{d c}\right\rangle$ with $\omega_{1}\left(\frac{d}{d a}\right)=1$ and $T \mathcal{L}=\operatorname{Ker} \eta \oplus\left\langle\frac{d}{d b}, \frac{d}{d c}\right\rangle$. Since $\mathrm{p}_{*}\left\langle\frac{d}{d b}, \frac{d}{d c}\right\rangle=\left\langle\frac{d}{d b}, \frac{d}{d c}\right\rangle$ (cf. (4.1)) and by (4.6), $\mathrm{p}_{*}: \operatorname{Ker} \omega_{1} \rightarrow T \mathcal{L}$ is an isomorphism. 


\subsection{Complex Contact Bundle on $\mathcal{L}$}

As $\mathbb{R}^{3}$ acts as translations on $\mathcal{M}, \mathbb{R}^{3}$ leaves $\omega$ (resp. $\omega_{i}(i=1,2,3)$ ) invariant. $\mathbb{R}^{3}$ induces the distribution of vector fields $\left\langle\frac{d}{d a}, \frac{d}{d b}, \frac{d}{d c}\right\rangle$ on $\mathcal{M}$. Define an almost complex structure $\bar{J}_{1}$ on $\operatorname{Ker} \omega_{1}$ as

$$
\bar{J}_{1} \mid \operatorname{Ker} \omega_{1}=J_{1}, \bar{J}_{1} \frac{d}{d b}=\frac{d}{d c}, \bar{J}_{1} \frac{d}{d c}=-\frac{d}{d b} .
$$

Lemma $4.1 \mathrm{p}_{*} \circ \bar{J}_{1}=J_{0} \circ \mathrm{p}_{*}$ on $\operatorname{Ker} \omega_{1}$.

Proof. Let $X \in \operatorname{Ker} \omega$. By the commutativity of (4.8)

$$
\mu_{*}\left(\mathrm{p}_{*}\left(J_{1} X\right)\right)=\mathrm{q}_{*} \nu_{*}\left(J_{1} X\right)=J_{0} \mathrm{q}_{*} v_{*}(X)=\mu_{*}\left(J_{0} \mathrm{p}_{*}(X)\right),
$$

so $\mathrm{p}_{*}\left(J_{1} X\right)=J_{0} \mathrm{p}_{*}(X)$.

Obviously, $\mathrm{p}_{*}\left(\bar{J}_{1}\left(\frac{d}{d b}, \frac{d}{d c}\right)\right)=J_{0} \mathrm{p}_{*}\left(\frac{d}{d b}, \frac{d}{d c}\right)$.

Lemma 4.2 $\bar{J}_{1}$ is integrable on $\operatorname{Ker} \omega_{1}$.

Proof. Let $\operatorname{Ker} \omega_{1} \otimes \mathbb{C}=T_{\omega_{1}}^{1,0} \oplus T_{\omega_{1}}^{0,1}$ be the eigenspace decomposition. Then $T_{\omega_{1}}^{1,0}=T_{\omega}^{1,0} \oplus\left\langle\frac{d}{d b}-\frac{d}{d c} \mathbf{i}\right\rangle$. If we note that $d \omega_{1}\left(\bar{J}_{1} X, \bar{J}_{1} Y\right)=d \omega_{1}(X, Y)\left(X, Y \in \operatorname{Ker} \omega_{1}\right)$ from (4.7), then $\left[T_{\omega_{1}}^{1,0}, T_{\omega_{1}}^{1,0}\right] \subset \operatorname{Ker} \omega_{1} \otimes \mathbb{C}$. Then $\mathrm{p}_{*}\left(\left[T_{\omega_{1}}^{1,0}, T_{\omega_{1}}^{1,0}\right]\right)=$ $\left[T^{1,0}(\mathcal{L}), T^{1,0}(\mathcal{L})\right]$. Since $J_{0}$ is the complex structure on $\mathcal{L},\left[T^{1,0}(\mathcal{L}), T^{1,0}(\mathcal{L})\right] \subset T^{1,0}(\mathcal{L})$. It follows

$$
\left[T_{\omega_{1}}^{1,0}, T_{\omega_{1}}^{1,0}\right] \subset T_{\omega_{1}}^{1,0} .
$$

Remark 4.3 The pair $\left(\operatorname{Ker} \omega_{1}, \bar{J}_{1}\right)$ is not a strictly pseudoconvex $C R$-structure on $\mathcal{M}$ unlike Sasakian 3-structures. For this, $\left[\frac{d}{d b}, \frac{d}{d c}\right]=0$ in $\operatorname{Ker} \omega_{1}=\operatorname{Ker} \omega \oplus\left\langle\frac{d}{d b}, \frac{d}{d c}\right\rangle$, so $d \omega_{1}\left(\frac{d}{d b}, \frac{d}{d c}\right)=0$. However, $d \omega_{1}: \operatorname{Ker} \omega \times \operatorname{Ker} \omega \rightarrow \mathbb{R}$ is nondegenerate from (4.7).

We put Ker $\eta \otimes \mathbb{C}=T_{\eta}^{1,0} \oplus T_{\eta}^{0,1}$. Let $\mathrm{p}_{*}: \operatorname{Ker} \omega_{1} \otimes \mathbb{C} \rightarrow T \mathcal{L} \otimes \mathbb{C}$ be an isomorphism so that $\mathrm{p}_{*}\left(\frac{d}{d b}-\frac{d}{d c} \mathbf{i}\right)=\frac{d}{d b}-\frac{d}{d c} \mathbf{i}$. By Lemma 4.1, we have $\mathrm{p}_{*}\left(T_{\omega}^{1,0}\right)=T_{\eta}^{1,0}$.

Theorem 4.4 The complex $2 n$-dimensional holomorphic subbundle $T_{\eta}^{1,0}$ is a complex contact subbundle on $\mathcal{L}$.

Proof. Let $T_{\omega_{1}}^{1,0} \otimes \mathbb{C}=T_{\omega}^{1,0} \oplus\left\langle\frac{d}{d b}-\frac{d}{d c} \mathbf{i}\right\rangle$ and $T^{1,0}(\mathcal{L})=T_{\eta}^{1,0} \oplus\left\langle\frac{d}{d b}-\frac{d}{d c} \mathbf{i}\right\rangle$ as above. From Remark 4.3, $d \omega_{1}: T_{\omega}^{1,0} \times$ $\bar{T}_{\omega}^{1,0} \rightarrow \mathbb{C}$ is nondegenerate. Since $J_{1}\left(J_{3} X\right)=-\mathbf{i}\left(J_{3} X\right), J_{3} X \in \bar{T}_{\omega}^{1,0}$. Then $d \omega_{1}\left(J_{3} X, Y\right)=-d \omega_{2}(X, Y)=\omega_{2}([X, Y])$ from (4.7). Thus $\omega_{2}\left(\left[T_{\omega}^{1,0}, T_{\omega}^{1,0}\right]\right)=\mathbb{C}$. In particular, $\left[T_{\omega}^{1,0}, T_{\omega}^{1,0}\right] \neq\{0\}$. As $\left[T_{\omega}^{1,0}, T_{\omega}^{1,0}\right] \subset T_{\omega_{1}}^{1,0}=T_{\omega}^{1,0} \oplus\left\langle\frac{d}{d b}-\frac{d}{d c} \mathbf{i}\right\rangle$ by Lemma 4.2 , it follows

$$
\left[T_{\eta}^{1,0}, T_{\eta}^{1,0}\right] \equiv\left\langle\frac{d}{d b}-\frac{d}{d c} \mathbf{i}\right\rangle \bmod T_{\eta}^{1,0}
$$

Hence $T_{\eta}^{1,0}$ is a complex contact subbundle on $\mathcal{L}$.

\section{References}

Alekseevsky, D., \& Kamishima, Y. (2008). Pseudo-conformal quaternionic $C R$ structure on ( $4 n+3)$-dimensional manifolds. Annali di Matematica Pura ed Applicata, 187(3), 487-529. http://dx.doi.org/10.1007/s10231-007-0053-2

Blair, D. E. (2002). Riemannian geometry of contact and symplectic manifolds Contact manifolds. Progress in Math., 203 Birkhäuser.

Foreman, B. (2000). Boothby-wang fibrations on complex contact manifolds. Differential Geom. Appl., 13, 179196. http://dx.doi.org/10.1016/S0926-2245(00)00025-5

Fried, D. (1980). Closed similarity manifolds. Comment. Math. Helv., 55, 576-582. http://dx.doi.org/10.1007/BF02566707 
Ishihara, S., \& Konishi, M. (1979). Real contact and complex contact structure. Sea. Bull. Math., 3, 151-161.

Kamishima, Y. (1999). Geometric rigidity of spherical hypersurfaces in quaternionic manifolds. Asian J. Math., 3(3), 519-556.

Kobayashi, S. (1959). Remarks on complex contact manifolds. Proc. AMS, 10, 164-167. http://dx.doi.org/10.2307/2032906

Kulkarni, R. (1978). On the principle of uniformization. J. Diff. Geom., 13, 109-138.

Lebrun, C. (1995). Fano manifolds, contact structures, and quaternionic geometry. Int. J. Math., 3, 419-437. http://dx.doi.org/10.1142/S0129167X95000146

Miner, R. (1991). Spherical CR-manifolds with amenable holonomy. International J. of Math., 1(4), 479-501. http://dx.doi.org/10.1142/S0129167X9000023X

Moroianu, A., \& Semmelmann, U. (1996). Kählerian Killing spinors, complex contact structures and twistor spaces. C.R. Acad. Sci. Paris, t. 323, Série I, 57-61.

Salamon, S. (1989). Riemannian geometry and holonomy groups. Pitman research notes in Math., 201, Longman Scientific.

Tanno, S. (1996). Remarks on a triple of $K$-contact structures. Tôhoku Math. Jour., 48, 519-531. http://dx.doi.org/10.2748/tmj/1178225296

Wolf, J. (1965). Complex homogeneous contact manifolds and quarternionic symmetric spaces. J. Math. and Mech., 14, 1033-1047.

\section{Copyrights}

Copyright for this articleis retained by the authors, with first publication rights granted to the journal.

This is an open-access article distributed under the terms and conditions of the Creative Commons Attribution license (http://creativecommons.org/licenses/by/3.0/). 